\title{
Study of Sensor Layout Acquisition and Application Analysis within Distribution Room
}

\author{
WU Bingxin ${ }^{1 *}$, HUANG Lijun ${ }^{1}$, WEI Yong ${ }^{1}$, LI Jungang ${ }^{1}$, ZHANG Jianyu ${ }^{1}$, MENG Le ${ }^{1}$, YIN Jianlun ${ }^{1}$, LIU Quanhang ${ }^{1}$ \\ ${ }^{1}$ (XJ Group Research and Development Center, Xuchang 461000, China)
}

\begin{abstract}
The traditional power distribution room lacks intelligent monitoring business functions, which is not conducive to the requirements of intelligent operation and maintenance. This article takes the intelligent IoT terminal(Internet of Things terminal) as the center, carries out the layout design of the distribution room sensor, combines the intelligent collection of the distribution room, and accesses the sensing equipment to build a smart distribution room, meanwhile, this paper designs the intelligent terminal which adopts the edge computing technology to carry out the environment temperature and humidity assessment, equipment partial discharge analysis and assessment, temperature rise assessment and other micro-applications to provide support for the construction of smart distribution room.
\end{abstract}

\section{Preface}

At present, most of the power distribution rooms in China have not implemented intelligent monitoring, and all equipment inspections and operations are carried out manually. The workload is large, and the inspection cycle is long, as well as the efficiency is low. It is impossible to analyze the operation of the distribution room in time. The situation restricts the further improvement of the equipment management level [1-5]. In recent years, the information and communication technology represented by the Internet of Things has gradually merged with the power distribution network to form the distribution internet of things [6], which provides solutions to many problems in the environment and equipment monitoring of the power distribution room. This paper uses the edge computing technology to construct the power distribution room. The edge IoT terminal is used as a data processing center to carry advanced business applications, and realize localized online monitoring, intelligent analysis and decision-making control of the operating status of the station under its jurisdiction. This paper studies and proposes the sensor layout of the distribution room and the typical application scheme of data services to guide the construction of the distribution room.

\section{Application Architecture based on IoT terminal}

The IoT terminal is used for the comprehensive monitoring of smart distribution room with voltage levels of $35 \mathrm{kV}$ and below, and realizes the monitoring of station environment monitoring, switchgear load operation monitoring, bus temperature monitoring, cable temperature monitoring, environmental monitoring, security monitoring, and other harmful gas online monitoring of SF6, 02, H2S, O3 meanwhile, controls the heater and ventilation, Lights, fans, dehumidifier. The IoT terminal follows the "cloud-tube-edge-end" IoT system and edge computing architecture, integrates the IoT gateway function, and supports the free expansion of business functions through application software (APP). The data collected by the IoT terminal is uploaded to the cloud platform through wireless/wired methods to realize the remote monitoring and equipment control of the station equipment and the optimization of the environment, meanwhile, it will avoid the malfunction of the distribution equipment caused by out of control in operation environment, which will ensure maintenance personnel safety, extend the service life of equipment, and reduce the problem of excessive cost caused by extensive management of the power distribution room, meanwhile, further improve the operation and management level of the station equipment. The IoT terminal sensor data monitoring architecture is shown in Figure 1.

\footnotetext{
* Corresponding author: wubingxincc@ $163 . c 0 m$
} 


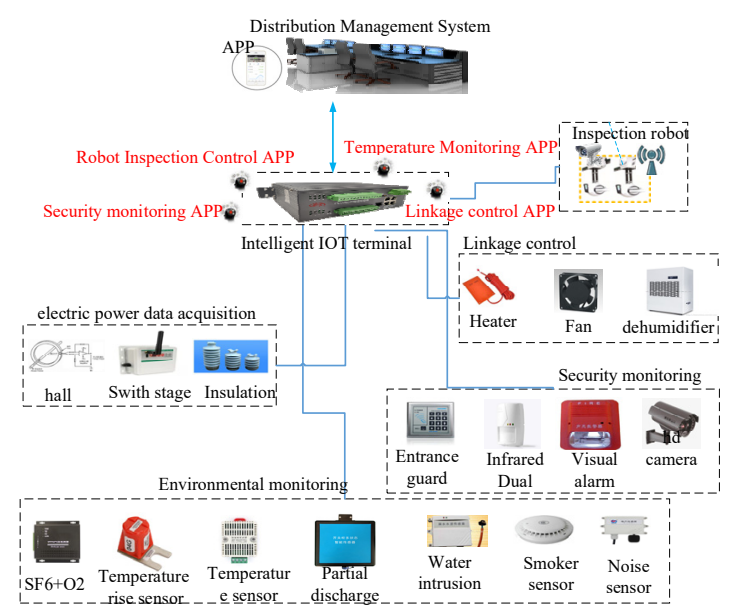

Fig.1. Intelligent monitoring architecture of the distribution room

As the "brain" of the distribution room, the cloud platform monitoring and management system is responsible for the storage, processing, and analysis of the station environment and equipment status information, and realizes online monitoring of station room panoramic data, video remote monitoring, multi-system/equipment linkage, and equipment electronic station accounts record and other functions; in addition, intelligent operation and maintenance applications for power distribution stations can be developed on the basis of data statistics and analysis. At the same time, it can also be used as a working platform for operation and maintenance personnel to realize remote monitoring, danger early warning and abnormal warning of the overall operating status of the distribution station room.

\section{Application of sensor layout in power distribution room}

The sensor layout of the power distribution room uses various sensor units to comprehensively monitor the sensor data in the distribution room, and the data is sent to the IoT terminal for unified on-site edge computing and management. The sensor information data include: 1) electrical equipment status information monitoring: including key equipment temperature monitoring, partial discharge monitoring, cable temperature monitoring, grounding switch monitoring, etc.2) environmental monitoring: flooding monitoring, environmental temperature and humidity monitoring, smoke monitoring, noise monitoring, and the linkage adjustment of dehumidifiers and air conditioner ; 3) Video security information monitoring: camera with infrared imaging、 access control and security linkage functions.

\subsection{Electrical equipment sensor data monitoring}

Considering the convenience of sensor installation and construction, the limitation of the space in the switchgear and other factors, the current sensor configuration of the electrical equipment in the station is mainly for the inlet and outlet connectors of dry-type transformer, busbar connectors, high-voltage and low-voltage switchgear. A temperature sensor is installed at the joint of each phase of the circuit breaker; each high-voltage switchgear is equipped with a partial discharge sensor, which is installed on the side wall of the cabinet. If the station has equipment containing SF6 gas, monitoring equipment will be placed near the equipment, and all the monitoring data will be uploaded to the IoT terminal.

\subsection{Temperature sensor}

The temperature sensor mainly measures the temperature of the terminals of key equipment in the power distribution room. When the temperature of the equipment exceeds the set threshold, an alarm message will be sent out by the IoT terminal.

\subsection{Partial discharge sensor configuration}

The partial discharge sensor mainly monitors the discharge of key equipment in the power distribution room to prevent greater accidents due to partial discharge. When the partial discharge occurs, an alarm message is issued by the IoT terminal.

\subsection{SF6 gas monitoring}

The SF6 gas monitoring sensor in the distribution room is connected to the IoT terminal through the RS-485 interface and the switch alarm cable. The control center can monitor the situation of the on-site SF6 equipment in real time. The data of indoor oxygen content and SF6 content is uploaded to the platform in real time, so that managers can check and find hidden dangers at any time; when indoor hypoxia or SF6 exceeds the standard, the IoT terminal will output a alarm signal to the operation and maintenance management system to remind the maintenance personnel to rush in time. When the IoT terminal detects that the SF6 gas exceeds the standard, the fan can be started and stopped to realize the automatic discharge of SF6 gas, so that the indoor oxygen content can return to the normal value; at the same time, the sound and light alarm equipment of SF6 outdoor is linked to remind the staff not to enter.

\section{Monitoring of the environmental information within distribution room}

\subsection{Temperature and humidity sensor}

Monitoring the temperature and humidity of the distribution room, when the area of the power distribution room $\mathrm{S}$ is $<75 \mathrm{~m}^{2}$, with 2 sensors; when $\mathrm{S}$ is $75 \sim 100 \mathrm{~m}^{2}, 3$ sensors; and when $\mathrm{S}$ is greater than $100 \mathrm{~m}^{2}$, 4 sensors; the sensors can be install on the opposite or back of the operation area of the power distribution cabinet, at a height of 1.2 to 1.4 meters from the ground, and avoiding installation near the outlet of the air conditioner and the radiation range of hot and cold wind. 


\subsection{Flood sensor}

If there is a basement, a flood sensor shall be installed on the entrance wall of the power distribution room. The water sensor probe is installed on the wall at a distance of $1 \sim 5 \mathrm{~cm}$ from the ground, and the detector is installed at a distance of more than 1 meter from the ground.

\subsection{Smoke detector}

The detector is installed on the roof of the power distribution room. When there is a fire hazard, it will send out an alarm signal, the sensor information will be connected to the IoT terminal, and the alarm will be activated in parallel. Each row of distribution cabinets is equipped with 1 3 smoke detectors on the upper part of the operating equipment, and the top of the power distribution room is ceiling mounted.

\subsection{Video security information monitoring}

Monitoring systems such as security and access control realize the monitoring of the operation in the distribution room, and at the same time realize the monitoring of the main equipment operation status. Through the video monitoring module, the operation and maintenance personnel can remotely view the operating status of the distribution room, and can remotely check the accuracy of the alarm information through the video monitoring equipment and make a reasonable maintenance plan in time

\section{5 loT terminal sensing data processing architecture}

IoT terminal is the core link of the power distribution internet of things system. It is located between the sensor device and the cloud platform. The edge computing node framework of the IoT terminal includes three layers: basic platform, information collection, and business application.

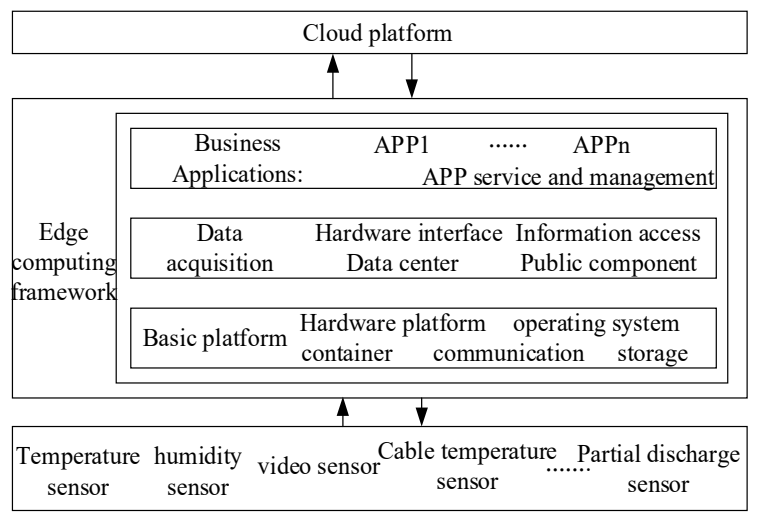

Fig.2. IoT terminal data processing architecture

The IoT terminal collects the electrical data, environmental temperature and humidity data, flooding data, fire protection data, video monitoring data, and other video data to realize the online monitoring, and ensures the safe, stable and reliable operation of the power distribution room. The intelligent IoT terminal adopts edge computing architecture of the Internet of Things, with hardware platformization and software APP function, and it can be flexibly configured with various application functions. APP software contains many applications, such as security monitoring APP, robot inspection control APP, linkage control APP, temperature control APP, etc., to meet the multi-service and reconfigurable functional requirements of the distribution room.

\section{Typical business applications of sensor data}

\subsection{Temperature and humidity control micro application}

Realize the collection and analysis of the temperature and humidity sensor data of the distribution room. When the temperature exceeds the fixed temperature, the IoT terminal will start the air conditioner to cool down and when the temperature drops to the set value, the air conditioner will be stopped in linkage. The humidity control logic is similar to the temperature control. The temperature and humidity logic control is shown in Figure 3.

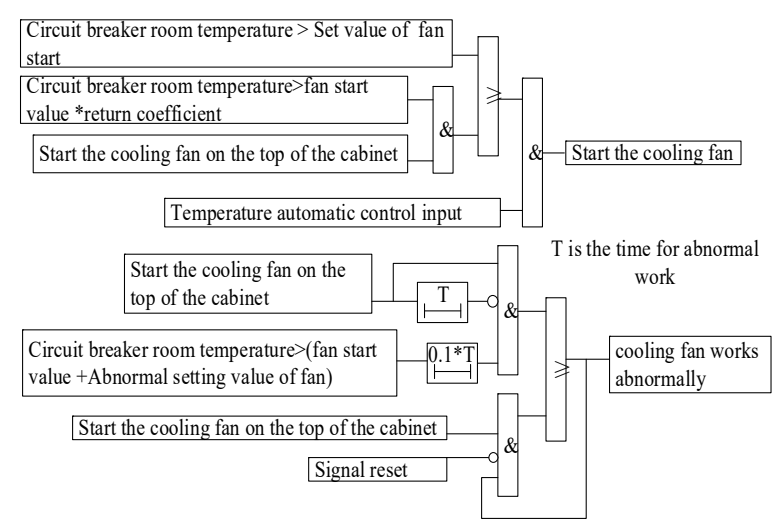

Fig.3.Temperature and humidity control logic schematic

\subsection{Switchgear mechanical life assessment micro application}

The IoT terminal statistical divide and close times of breaker, which contains handcar breakers and ground switch breakers, it then compares with the number of specified mechanical life times. When the proportion reaches $95 \%$, a warning signal will be sent by IoT terminal to support equipment operation services. 


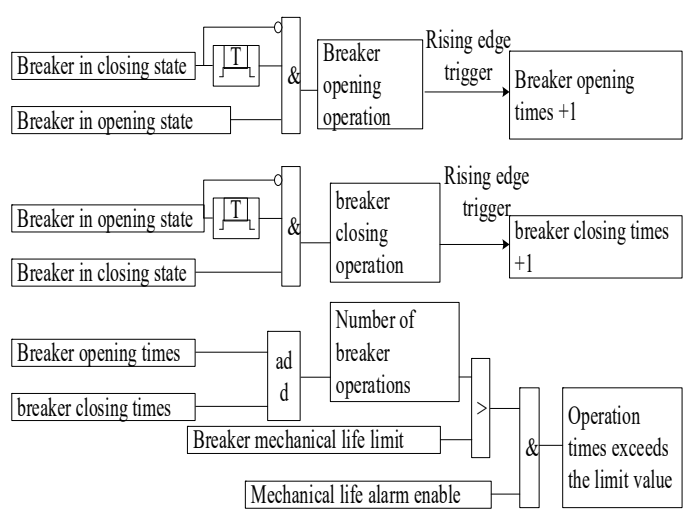

Fig.4. Micro-application logic schematic of breaker mechanical life analysis

\subsection{Partial discharge monitoring and evaluation micro application}

The IoT terminal collects partial discharge equipment information, analyzes and evaluates the attention state, abnormal state, serious state, and normal state, and supports the intelligent operation and maintenance of the switchgear.

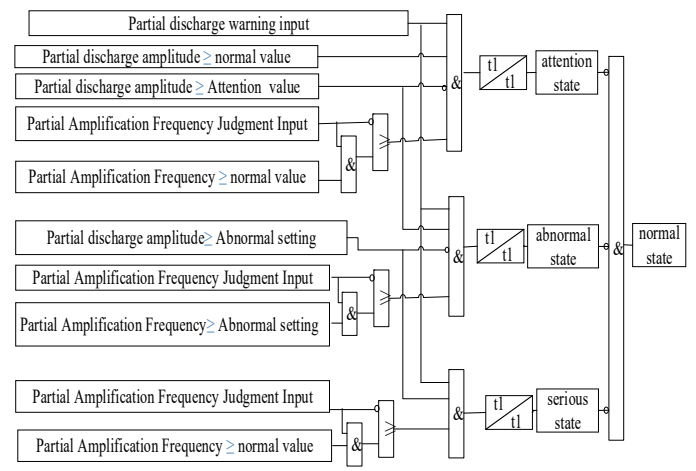

Fig.5.Switchgear partial discharge micro application logic schematic

\subsection{Micro-application of temperature rise assessment}

The temperature rise assessment includes the upper and lower three-phase contacts of breaker, the temperature of the cable head, and the busbar temperature.

The temperature rise difference are designed as four tap positions, for example, $[0 \mathrm{~K} \sim \mathrm{T} 1]$ is normal state; [T1 T2] is defined as the attention state; [T2 T3] is abnormal state; [T3 $\sim \mathrm{K}$ ] is defined as severe state. In general, the setting values of the three fixed values are respectively taken as $4 \mathrm{~K}, 8 \mathrm{~K}$, and $12 \mathrm{~K}$.

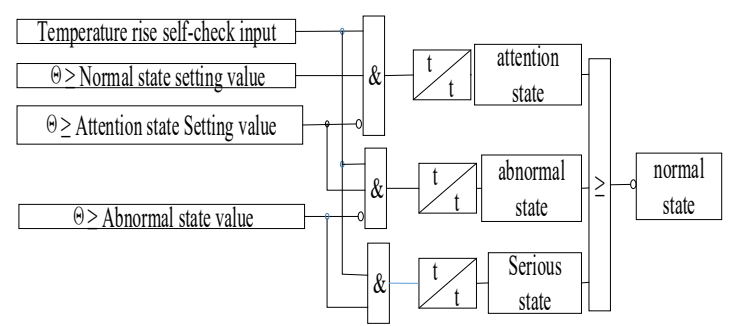

Fig.6.Temperature rise assessment micro application logic schematic

\section{Conclusion}

Under the development of the internet of things in the power distribution room, this article takes the IoT terminal as the center, and at the same time, builds the Internet of Things system in the distribution room based on the intelligent sensor collection and the access of the sensing equipment. It also designs the sensor layout of the distribution room and the data access and transmission method of the IoT terminal, and provides the micro-application design for the analysis and evaluation of the temperature and humidity of environment and equipment, the analysis and evaluation of the partial discharge of typical equipment, and the evaluation of the equipment temperature rise. It will provide support for the construction of smart distribution room, meanwhile, the sensor data will be effectively processed and utilized, furthermore, this method will give the support and guide to improve the operation, maintenance and management level of the power distribution room.

\section{References}

1. LÜ Jun, SHENG Wanxing, LIU Riliang, et al. Design and application of power distribution Internet of Things[J]. High Voltage Engineering, 2019, 45(6): 1681-1688

2. HUI Xiaolin, SUN Zhenquan. Integration of intelligent distribution network and internet of things[J]. Internet of Things Technologies, 2011, 1(8): 31-35.

3. LIU Riliang, LIU Haitao, XIA Shengfeng, et al. Internet of Things technology application and prospects in distribution transformer service area management[J]. High Voltage Engineering, 2019, 45(6):1707-1714.

4. SUN Haoyang, ZHANG Jichuan, WANG Peng, et al. Edge Computation Technology Based on Distribution Internet of Things[J]. Power System Technology, 2018, 42(10): 3110-3117.

5. NAGI J, YAP K S, NAGI F, et al. NTL detection of electricity theft and abnormalities for large power consumers In TNB Malaysia[C]//2010 IEEE Student Conference on Research and Development (SCOReD). December 13-14, 2010, Putrajaya, Malaysia. IEEE, 2010: 202-206.

6. ZHANG Dongxia, WANG Jiye, LIU Keyan, et al. Application of big data technologies in power distribution and utilization system[J]. Distribution \& Utilization, 2015, 32(8): 6-11. 